\title{
Low-Field NMR of Water in Model Soils
}

\author{
Oscar Sucre $^{*}, 1$, Federico Casanova ${ }^{1}$, Andreas Pohlmeier ${ }^{2}$ and Bernhard Bluemich ${ }^{1}$
}

\author{
${ }^{1}$ ITMC RWTH-Aachen University, Aachen, D-52056, Germany \\ ${ }^{2}$ Forschungszentrum Jülich, Jülich, D-52425, Germany
}

\begin{abstract}
The evolution of water contained in soils is a physical phenomenon of importance in soil science and climatology. This work presents preliminary results from the use of mobile NMR to measure moisture in soil columns. To demonstrate the ability of the NMR technique to follow the drainage process of water in model soils, moisture measurements were performed at a certain depth with a mobile NMR sensor during an one-step outflow experiment. The NMR sensor exhibits a cylindrical geometry incorporating the principle of the u-shaped NMR-MOUSE. It could be raised and lowered inside a plastic tube in the soil column similar to a wire-line logging tool. Working with a frequency of $8.8 \mathrm{MHz}$, the sensitive volume lies $3 \mathrm{~mm}$ deep into the soil and $2 \mathrm{~mm}$ away from the tube walls, making the technique truly non-invasive. For the purpose of quantitative analysis, the temporal evolution of moisture (described by the Richards Equation) was modelled with the Hydrus1D Program. Out of these simulations, an assessment of the hydraulic parameters (Ks, $\alpha, \mathrm{n}$ of the Van Genuchten model) of the soil was achieved.
\end{abstract}

Keywords: Low field NMR, moisture sensor, model soils, drainage, richards equation.

\section{INTRODUCTION}

The question of how water moves in soils is of importance in agriculture and climatological sciences.

Being a partially saturated porous medium, soil in the vadose zone can either stop or permit the transport of water depending on gravity, surface tension and evaporation. Probing of moisture on field plays a central role in addressing these issues, and several techniques have gained acceptance in the soil science community to perform that measurement: Time Domain Reflectometry (TDR) [1] and Neutron Absortion [2] among others.

The application of Nuclear Magnetic Resonance (NMR) in soils is not new, as it has been applied to gain information about the pore size distribution [3, 4], in the search of aquifers [5] or to study the chemistry of soil organic matter [6, 7]. However as far as we know, little has been done to develop an NMR instrument that can be deployed on field to measure local values of soil moisture, taking advantage of the non-destructive nature this technique possesses. In this sense, NMR at low fields has just begun to make its way among the above mentioned techniques. It can even show some comparative advantages over TDR: As the signal to probe water comes from the atomic nuclei (in case of water, simply protons), it is not affected by any ions in solution, so the NMR technique happens to be immune to any salt content in water absorbed in soil. Furthermore, due to the resonance condition and the inhomogeneous magnetic fields generated the sensor senses water in a very well defined volume [8], making the measure well resolved spatially. For those reasons we believe the NMR can become standard to

*Address correspondence to this author at the ITMC RWTH-Aachen University, Aachen, D-52056, Germany; Tel: 00-49-241-8026423;

E-mail: osucre@mc.rwth-aachen.de sense moisture among soil scientist. It has even reached a mature stage in its development as a logging tool for the oil industry $[9,10]$. Undeniably NMR is no excepted from experimental diffculties: low inherent signal-to-noise ratio constitutes its main drawback. Common way to improve this ratio is to average over repeatedly acquired data, however that can make the whole profiling of soil moisture costly in terms of time.

This article reports progress in probing soil moisture by low field NMR. The work was performed as a part of the interdisciplinary project Transregio 32 [11] (funded by the German Research Council DFG): the drainage processes in a first column filled with FH31 model soil and in a second column with W3 model soils under known boundary conditions were investigated with a newly developed low-field NMR sensor: the slim-line NMR logging tool. The moisture profile before and after drainage, and the moisture loss at certain depth during drainage were thus recorded with this instrument. To validate the results, the associated drainage problem is solved numerically, producing a theoretical curve that can be compared to the experimental results. By changing soil hydraulical parameters in the numerical solution the better correspondence between theory and experiment is found and the so obtained values are compared to reported in the literature.

\section{EXPERIMENT AND MATERIAL}

The experimental setup is shown in Fig. (1) and consists of two concentrical tubes, being the space between them filled with the model soil to research. This arrangement or soil "column" stays in a water tank. A copper fabric was wound around the outer tube to shield it from electromagnetic noise. The central inner column has a diameter of 31 $\mathrm{mm}$ and walls $1 \mathrm{~mm}$ thick. The NMR sensor moves within the inner tube. Initially the model soil is completely saturated by raising the water level in the tank up to top of the column. 
The top of the column is then closed with a rubber seal that allows the entrance of air (necessary for pressure balance during drainage) and limits evaporation as well. The bottom plate is connected to the water tank through perforations 4 $\mathrm{mm}$ wide and covered by a sheet of filter paper. This lets the water drain out from the column and gives hold to model soil lying thereupon. To obtain a complete profile of the saturation along the column the sensor is displaced with a stepper motor from the bottom to the top while NMR signal is acquired.

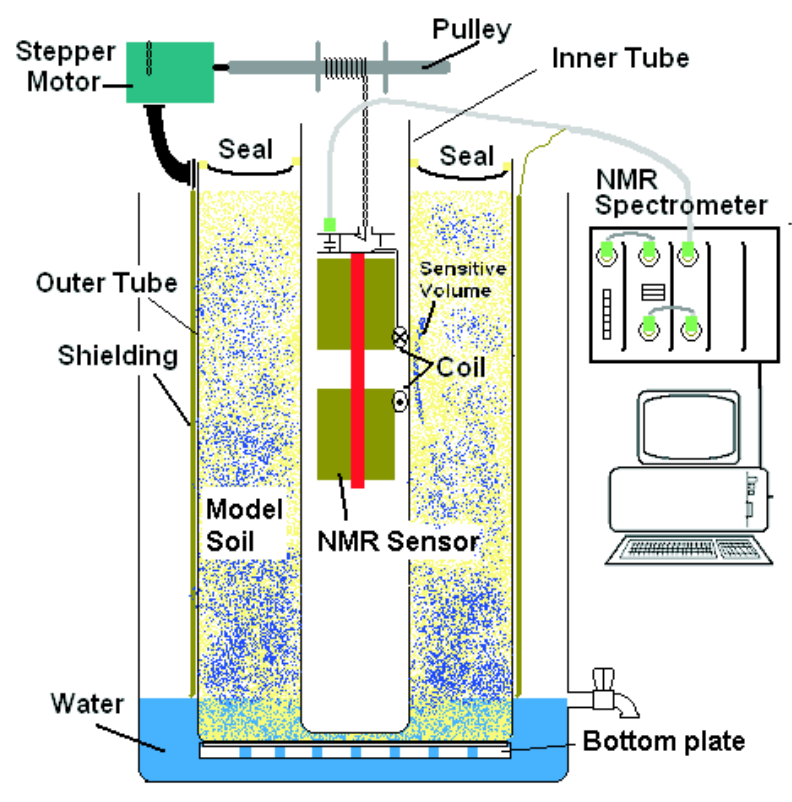

Fig. (1). Setup for soil drainage experiments.

Two experiments of drainage were performed, one on coarse sand FH31 (distribution of grain size classes 2\% (>0.72mm), 8\% (0.71-0.5mm), 30\% (0.5-0.355mm), $41 \%$ $(0.36-0.25 \mathrm{~mm}), 16 \%(0.25-0.18 \mathrm{~mm}), 3 \%(<0.18 \mathrm{~mm}))$ and the other on fine sand W3 (distribution of grain size classes $7 \%$ (0.6-0.2 mm),60\% (0.2-0.063 mm), 20\% (0.063-0.02 $\mathrm{mm}), 6 \%(0.02-0.0063 \mathrm{~mm}), 3(0.0063-0.0002 \mathrm{~mm}), 2 \%$ (< $0.002 \mathrm{~mm})$ ). Both obtained in Quartzwerke Frechen, Germany. Relevant parameters in these experiments are the corresponding column height, measurement time and the sample porosity $\theta_{S}$. They are summarized in Table 1 .

Table 1 Sample Porosities and Experimental Parameters

\begin{tabular}{|c|c|c|c|}
\hline Sample & Height (cm) & Time (min) & $\boldsymbol{\theta}_{\boldsymbol{S}}$ \\
\hline \hline FH31 & 67 & 1372 & 0.36 \\
\hline W3 & 59 & 14400 & 0.36 \\
\hline
\end{tabular}

After having reached complete saturation, the columns were let to drain by allowing the water to flow out of the tank. In the special case of FH31, the initial fall of the water level in the tank was recorded in time (Fig. 2). This is because the duration of the drainage process in FH31 (about 90 $\mathrm{min}$ ) is comparable to time the tanks needs to release all wa- ter (15 min) setting a boundary condition that must be taken into account for modelling. Afterwards the water is kept at the same level during the rest of the experimental time. Due to the fast drainage in FH31, moisture profiles were acquired before and after the drainage only.

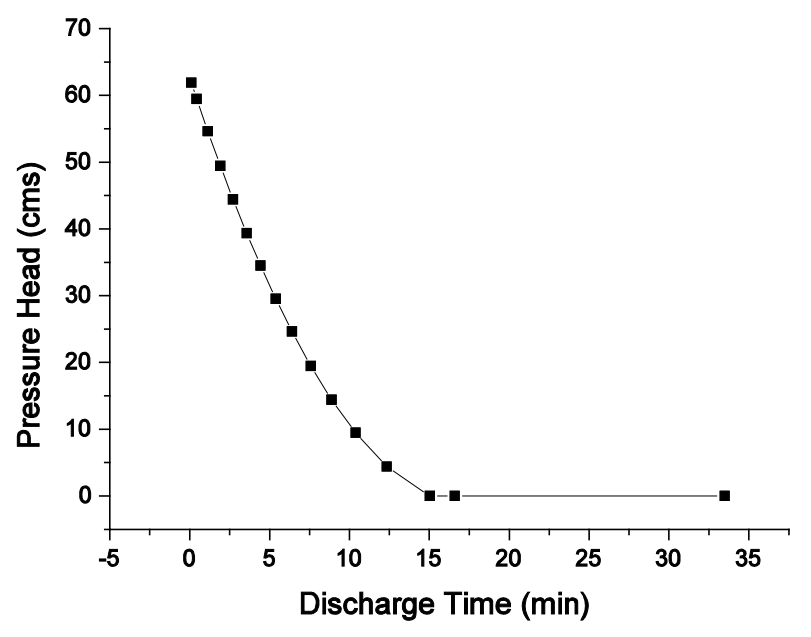

Fig. (2). Pressure head at bottom outside the column.

Another important aspect in the experiment for FH31 is the drainage dynamics. Since the drainage occurs so fast in comparison to the time needed to perform a sole moisture profile, we decided to follow it by maintaining the sensor at a depth of $-22.1 \mathrm{cms}$ during the first $150 \mathrm{~min}$ of experimental time. At this depth, following previous moisture profiles, we are certain of sensing a considerable drop in moisture. Although this corresponds to read the water content at a sole point, the data so obtained contains enough information to characterize the temporal behavior of the drainage completely. Here the number of NMR acquisitions per moisture measurements (scans) plays a critical role: It must be high enough to guarantee a small uncertainty (say 7\%) but it should be made in a time suficiently short to follow reliably the temporal change in water saturation. In our case, given the NMR longitudinal relaxation time of water in our sand $\left(T_{1}=800 \mathrm{~ms}\right)$ and a signal-to-noise ratio per echo and per scan of 0.17 (in FH31), 64 scans with 128 echoes were enough to achieve the aimed uncertainty $(7 \%)$ at each point. In the case of W3 a moisture profile of the water content was measured each two days.

\section{INSTRUMENT AND DATA PROCESSING}

Initially perceived as a potential tool for medical research [14], the NMR slim-line logging tool has been built with a cylindrical shape. It is an inside-out NMR instrument similar to some others found in the literature [12]. Fig. (3) shows a picture of the sensor. Generally speaking, conditions to observe the nuclear resonance are a constant magnetic field $\vec{B}_{0}$ and a radiofrequency (rf) field $\vec{B}_{1}$. The latter is generated by a coil and the former by a magnet body, establishing together a sensitive volume about $3 \mathrm{~mm}$ above the coil (Fig. 4). A sequence of pulses is applied in the rf field, exciting therefore the spins in resonance. Since the inner tube where the sensor is displaced is only $1 \mathrm{~mm}$ thick, the sensitive volume is $2 \mathrm{~mm}$ well inside the soil, avoiding disturbance in the 


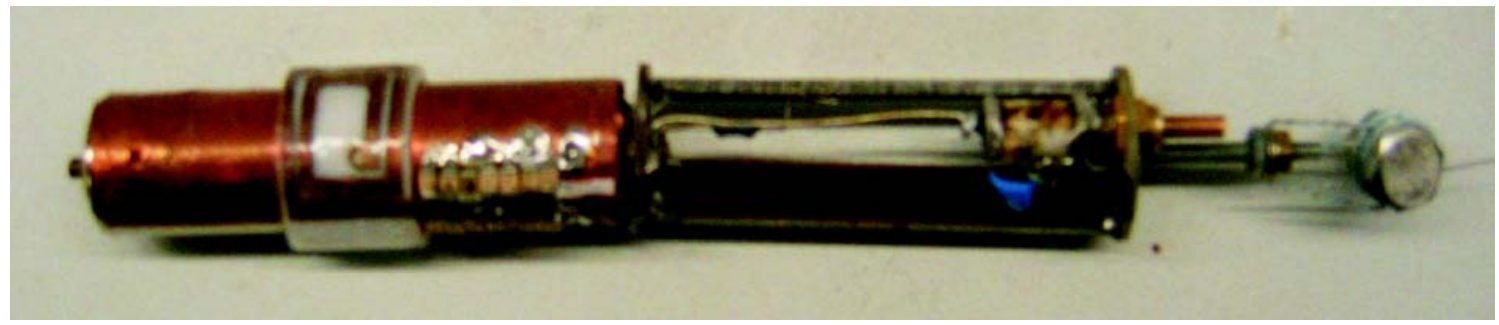

Fig. (3). Photo of the slim-line NMR soil sensor.

measurement due to any possible overlapping between the walls and the sensitive volume. This depth of the sensitive volume was experimentally checked by measuring the NMR signal of rubber layers of different thickness. The main body of the sensor consists of two cylindrical magnets $8 \mathrm{~cm}$ long each (1 in the Fig. 4) and $3 \mathrm{~cm}$ in diameter (D) and with a magnetization $\vec{M}$ pointed as depicted, separated by a gap $\mathbf{g}$ of $4 \mathrm{~mm}$. Just above the gap lies the $\mathrm{rf}$ coil (c). The sensitive volume is around $30 \mathrm{~mm}^{3}$, being its position and thickness determined by profiles of the magnetic fields $\vec{B}_{0}$ and $\vec{B}_{1}$, the operation frequency $(8.8 \mathrm{MHz})$, the local value of the magnetic gradient $(30 \mathrm{~T} / \mathrm{m})$ and the pulse length used (6 $\mu \mathrm{sec})$ in the pulse sequence [8]. The coil has a dead time of $20 \mu \mathrm{sec}$ approximately. The NMR spectrometer used was a Minispec Serie $m q$ from Bruker.

In low field applications, the NMR signal is usually acquired with a Carr-Purcell-Meilboom-Gill (CPMG) [12] pulse sequence, with pulses separated by a echo time $\tau_{E}$. The succession of NMR echoes, sequentially acquired, compose altogether a decaying signal with a effective transverse relaxation time (here simply denoted by $T_{2, \text { eff }}$ ). Its decay under highly inhomogeneous magnetic fields is governed by translational diffusion of molecules, interactions among nuclear spins (the proper transversal relaxation) and interactions between the surface of the solid matrix and the nuclear spins [13]. In spite of this variety of relaxation mechanisms, averaging over a number of echoes $\left(N_{E}\right)$ still yields a figure directly proportional to water content, provided the time span that corresponds to these echoes $\left(\tau_{E} N_{E}\right)$ is small compared to $T_{2, \text { eff }}$. This can be easily shown by describing the succession of echoes with these following formulae, being $\mathrm{j}$ an index that labels the echo number (between the 1 and $N_{E}$ ), n(j) the noise at the echo $\mathrm{j}$ and $A_{1}$ the amplitude of the initial echo:

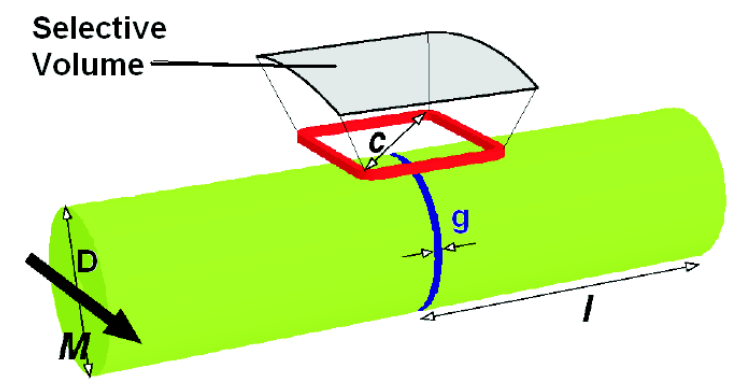

Fig. (4). Main parts of the slim-line NMR logging tool. M labels the magnetization vector.

$$
S(j)=A_{1} e \frac{-(j-1) \tau_{E}}{T_{2}, e f f}+n(J)
$$

If we average over the first $N_{E}$ echoes and apply well known mathematic formulae, we obtain $\langle S\rangle$ :

$$
<S>=\frac{A o}{N E}\left(\frac{e \frac{-(N E) \tau_{E}}{T_{2}, e f f}-1}{e \frac{-\tau_{E}}{T_{2}, e f f}-1}\right)+<n>N_{E}
$$

As long as $N_{E} \tau_{E}<0 * * 3 T_{2, e f f}$, we can approximate the exponential term to a linear function $\left(e^{-x} \approx 1-x\right)$ with an uncertainty of $5 \%$. Easy manipulations will lead to the following equation:

$$
<S>=A o+<n>N_{E}
$$

So it is proven that averaging over the first $N_{E}$ echoes has no further effect on the obtained data than averaging the random noise. This way of data processing has the advantage that for a complete signal of $N_{E}$ echoes, we diminish the noise amplitude to that we had if the experiment were repeated NE times. When acquiring noisy signals, this procedure is of not little advantage.

To give an example of this statement with real numbers, consider the Fig. (5) where data for water in saturated sand is depicted as it is supplied by the spectrometer. Experimental parameters in this decay are 256 scans, an echo time of $50 \mu \mathrm{s}$

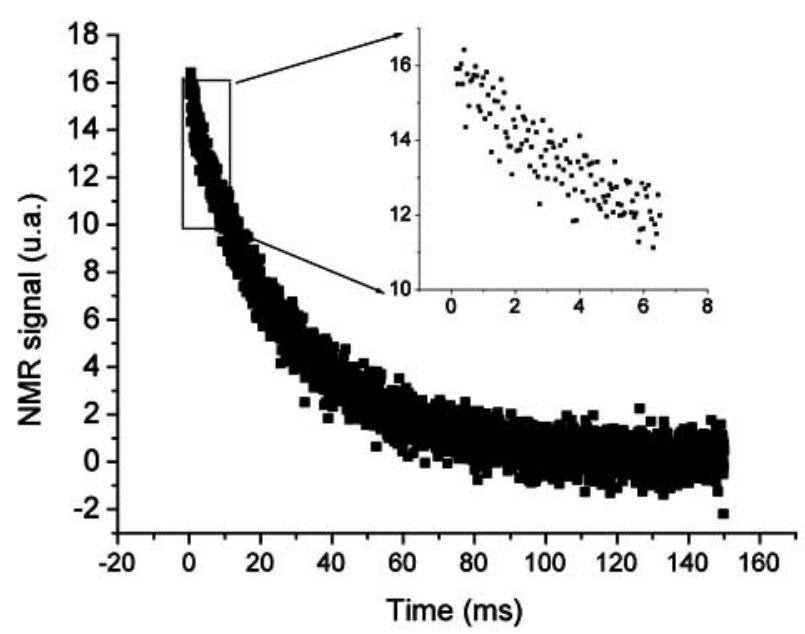

Fig. (5). NMR signal acquired in saturated sand FH31. $T_{2, \text { eff }}=28$ ms. 
and 3000 echoes. With a exponential function fitted to this whole data, an effective $T_{2, \text { eff }}$ of $28 \mathrm{~ms}$ can be easily obtained. The inset of this figure shows the first 128 echoes, corresponding to a time of $6.4 \mathrm{~ms}$, a time lower than the boundary of $30 \%$ set by the demonstration given above. So we can confidently average this first 128 echoes to obtain a relative measure of the partial saturation in sand. With prior knowledge of the signal level for completely saturated sand, the partial saturation in soil $\theta$ can then be calculated by a linear interpolation. This was the way of processing data for all the moisture profiles presented here.

\section{RESULTS}

The evolution of water saturation as a function of depth is shown in the Figs. (6 and 8) for the sand and the silt columns, respectively. A fact easy to recognize is the big difference in time evolution: while the FH31 has reached its equilibrium state in 1.5 hours, W3 does not show any important difference in saturation during 10 days.

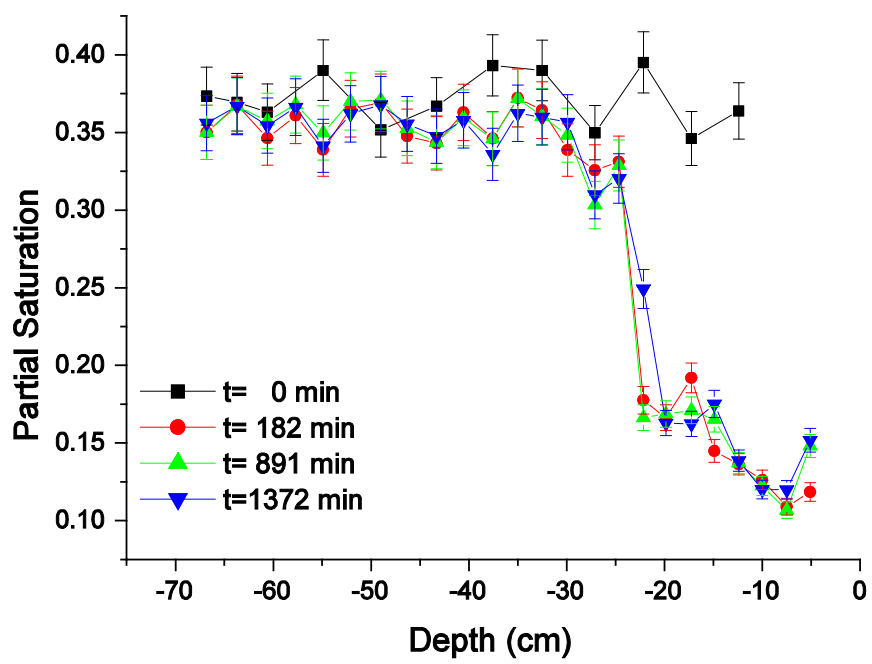

Fig. (6). Moisture profle in a column of model soil FH31. Height $=67 \mathrm{~cm}$.

Four profiles were measured in FH31, one prior to drainage $(\mathrm{t}=0$ min, Fig. 6) and three thereafter $(t=182,891$, $1372 \mathrm{~min}$ ). Experimental parameters for these measurements were: Echo time: $50 \mu \mathrm{sec}, 256 \mathrm{scans}, 3 \mathrm{sec}$ of recycling delay and 128 echoes. The similarity of these three last profiles reveals that the equilibrium state of the column had been reached already at the time of the first measurement (182 $\min )$. As is well known in soil physics, the profile in this state corresponds to the retention curve $\theta(h)$ [16]. We used therefore the profile at $\mathrm{t}=891 \mathrm{~min}$ for fitting of the retention curve.

For the dynamical measurement, done by leaving the sensor measuring at certain depth during drainage as it was explained before, the results are depicted as open symbols the Fig. (7). Experimental parameters were the same as for the moisture profiles with the sole exception of the scans: 64 instead of 256, for reasons already explained. The continuous curve correspond to the numerical solution for permeability at complete saturation $K_{s}$.

The situation with the model soil W3 was completely different. It retained water during 10 days with no measurable

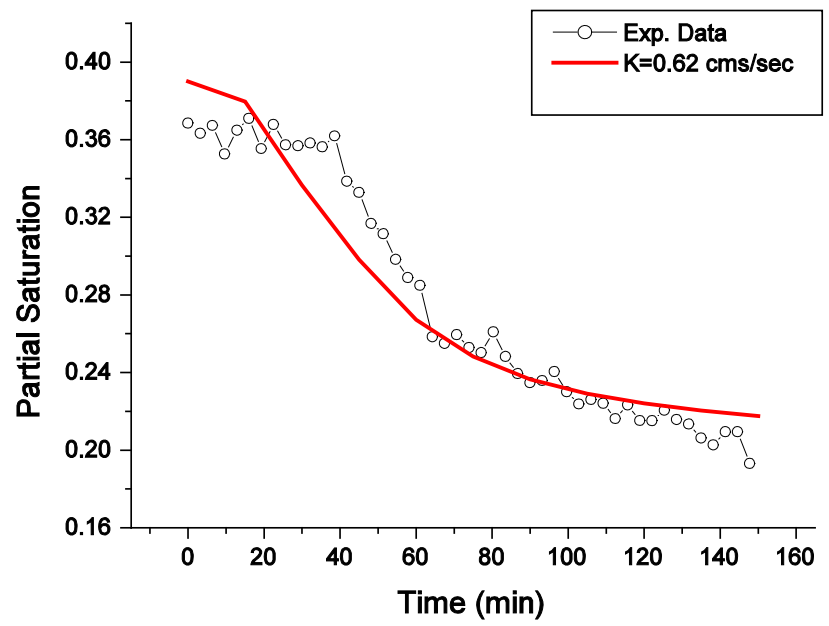

Fig. (7). Moisture drop at depth $-22.1 \mathrm{~cm}$ during $150 \mathrm{~min}$ for FH31. In color, numerical solution for $K_{s}=0.62 \mathrm{~cm} / \mathrm{sec}$.

change. Experimental parameters were the same than for FH31, although the used NE for averaging was 64 instead of 128 , as the $T_{2, \text { eff }}$ for this soil was $20 \mathrm{~ms}$. Given the height of the column the retention curve of this soil cannot be established (Fig. 8). However, a slight drop of 5\% from the value at the bottom can be observed along the profile, corresponding probably to the water loss in the biggest pores.

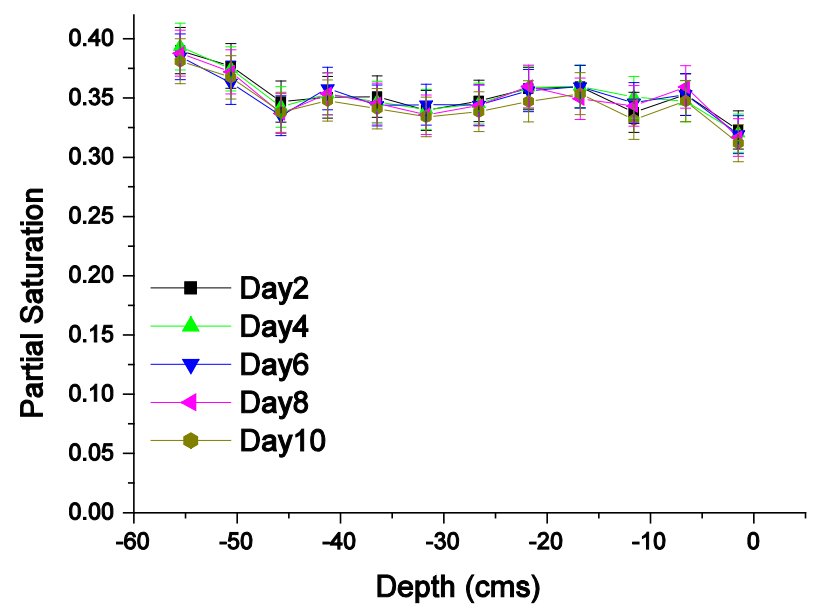

Fig. (8). Moisture profile in a column of model soil W3. Height=59 $\mathrm{cm}$.

\section{MODELING WITH RICHARDS EQUATION FOR THE FH31 COLUMN}

To validate the measurements obtained with this new method to probe soil moisture, we use the well known Richards equation (eq. 1) $[15,16]$. It establishes the theoretical framework to model the evolution of moisture profile in the sand column, where $h$ is the hydraulic head, $\theta$ the partial saturation, and $K_{s}$ the permeability of the soil at complete saturation. After starting the drainage process the level of the water mirror that initially was at the top of the column recedes completely to the bottom in about 15 min, setting a variable pressure head (Fig. 2) at the bottom. This data was 
taken as lower boundary condition for a numerical solution of eq. 1 .

$$
\frac{\partial \theta}{\partial t}=\frac{\partial}{\partial x}\left[K(\theta)\left(\frac{\partial h}{\partial x}+1\right)\right]
$$

To undertake the simulation of the drainage problem, we use the Van Genuchten Model [17] for the retention curve (eq. 3) (where $\alpha$ and $n$ are the Van Genuchten parameters) and the permeability in dependency of the relative saturation (4), having $K_{s}$ the meaning explained in the previous section. Parameter m equals $1-\frac{1}{n}$ and $1=0.5$ for most soils [15]. With the relative saturation $S_{e}$ defined by eq. 2 in terms of the real saturation $\theta$, the porosity $\theta_{s}$ and the residual saturation $\theta_{r}$; we obtain a complete set of equations to solve eq. 1 . The HYDRUS code [18] supplies the computational tool to perform 1D simulations to model the illustrated drainage process.

Several forward simulations were carried out to find the values of the Van Genuchten parameters $\alpha$ and $n$ that would reproduce the measured data. The best fit was corresponded to $\alpha=0.0268 \mathrm{~cm}^{-1}$ and $n=9.0$. The numerical profile and the experimental data are depicted in Fig. (9).

$$
\begin{aligned}
& S e=\frac{\theta-\theta}{\theta_{s}-\theta r} \\
& S e=\frac{1}{\left[1+(\alpha h)^{n}\right]^{m}} \\
& K\left(S_{e}\right)=K_{s} S_{e}^{l}\left[1-\left(1-S e^{\frac{1}{m}}\right)^{m}\right]^{2}
\end{aligned}
$$

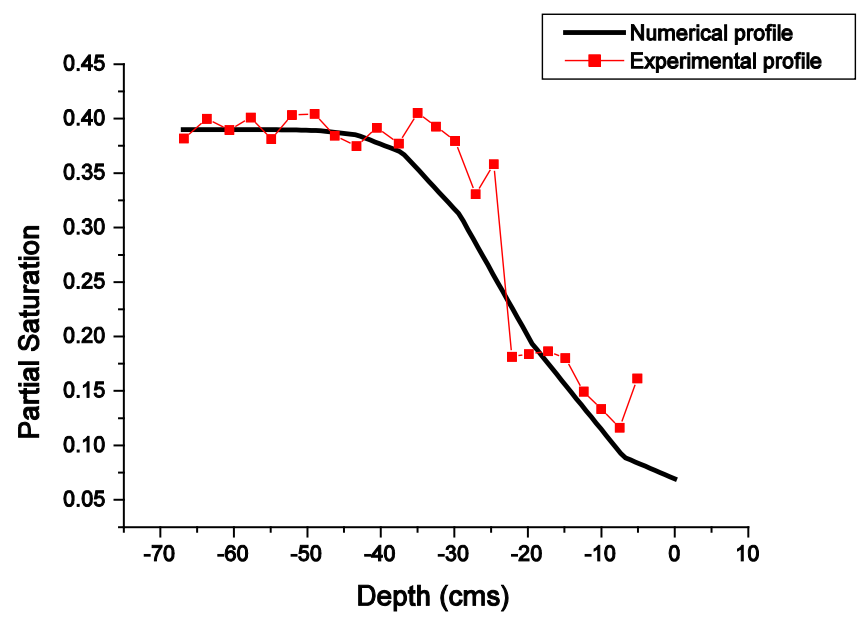

Fig. (9). Moisture profile in FH 31 at $\mathrm{t}=891 \mathrm{~min}$ and theoretical profile using the Van Genuchten model. $\alpha=0.0268 / \mathrm{cm}^{-1}$ and $n=$ 9.0 .

With these gained values for $\alpha$ and $n$, further simulations were performed with several values of the permeability at full saturation $K_{s}$, using the temporal change in partial saturation measured at a depth of $-22.1 \mathrm{~cm}$ for comparison (Fig.
7). Best fit among the numerical and the experimental curve was found for $K_{s}=0.62 \mathrm{~cm} / \mathrm{min}$.

\section{CONCLUSION AND OUTLOOK}

The instrument has shown its capability of following the fast drainage process in model soil (FH31). The so obtained data can be analyzed to extract hydraulical parameters of the model soil. However, experimental parameter like repetition time (recycling delay), number of scans and number of echoes must be carefully selected to follow the drainage process successfully. One interesting remark can be made when examining Fig. (7): There is some small delay in the moisture drop that the simulation does not reproduce, perhaps due to the hysteresis behavior in the retention curve of sand.

What information can be won of this information is still an open question, however we can see that small features in the temporal evolution of moisture can be followed with this instrument. About the Van Genuchten parameters obtained for FH31, that Kastelanek reports for a sand fraction with a grain sizes between 150 and $300 \mu \mathrm{m}$ a value of $n=8.35$ [19]. The obtained permeability corresponds to $80 \%$ of the value previously reported [20], measured with Darcy's Law. The values agree satisfactorily with literature data for a fine sand of the textural composition like ours, and thus prove the validity of the NMR sensor.

Work in progress concerns the construction of a sensor with a larger diameter of $48 \mathrm{~mm}$ that allows to measure 6 $\mathrm{mm}$ inside the soil at a frequency of $12 \mathrm{MHz}$. Our short term goal is to reach a signal to noise ratio per scan and per echo of 18, similar of the NMR-MOUSE [12] at the same depth. This would in principle enable us to measure moisture profiles in shorter time, to avoid any surface effects due to wall proximity and to essay external noise suppression techniques, opening the way to a robust instrument which could be deployed outdoors.

\section{ACKNOWLEDGEMENTS}

One of the authors (Sucre) thanks the German Academic Exchange Service (DAAD) for his Ph. D. grant. Support by the Deutsche Forschungsgemeinschaft through research project TR32 is also grate fully acknowledged.

\section{REFERENCES}

[1] Robinson DA, Jones SB, Wraith JM, Or J, Friedman FP. A review of advances in dielectric and electrical conductivity measurement in soils using time domain reflectometry. Vadose Zone J 2003; 2: 444-75.

[2] Strebel DE, Landis DR, Huemmrich KF, Meeson BW, Eds. Collected data of the first ISLSCP field experiment, NASA Goddard Space Flight Center: Greenbelt, Maryland, USA 1988; Vol. 1.

[3] Hinedi ZR, Kabala ZJ, Skaggs TH, Borchardt DB, Lee RW and Chang AC. Probing soil and aquifer material porosity with Nuclear Magnetic Resonance. Wat. Res. Res 1993; 29: 3861-6.

[4] Pohlmeier A, Haber-Pohlmeier S, Stapf S. A fast field cycling nuclear magnetic resonance relaxometry study of natural soils. Vadose Zone J 2009; 8: 735-42.

[5] Meju M, Denton P, Fenning P. Surface NMR sounding and inversion to detect ground-water in key aquifers in England: comparisons with VESTEM methods. J Appl Geophys 2002; 50: 95-111.

[6] Conte P, Spaccini R, Piccolo A. O-alkylation of a lignite humic acid by phase-transfer catalysis. Anal Bioanal Chem 2006; 386: 382-90. 
[7] Jaeger F, Grohmann E, Schaumann GE. 1H NMR Relaxometry in natural humous soil samples: insights in microbial effects on relaxation time distributions. Plant Soil 2006; 280(1-2): 209-22.

[8] Balibanu F, Hailu K, Eymael R, Demco D, Bluemich B. Nuclear magnetic resonance in inhomogeneous magnetic fields. J Magn Reson 2000; 145: 246-58.

[9] Sezginer A, Goswami J, Luong B. On the design of NMR sensor for well-logging applications. IEEE Trans Antennas Propag 2000; 48: 1393-403.

[10] Kleinberg R. Well logging. In: Grant DM, Harris RK, Eds. Encyclopedia of nuclear magnetic resonance. Wiley: New York 1996; Vol. 8: p. 4960.

[11] Interdisciplinary Collaborative Research Center TR32. Patterns in the soil-vegetation-atmospheric system: monitoring, modeling, data assimilation. Funded by the Deutsche Forschungsgemeinschaft, DFG. 21, Jan, 2010, Available from: www.tr32.de

[12] Bluemich B, Perlo J, Casanova F. Mobile single-sided NMR. Prog Nucl Magn Reson Spectrosc 2008; 52: 197-269.

[13] Huerlimann M, Grffin D. Spin dynamics of carr-purcell-meiboomgill-like sequences in grossly inhomogeneous $B o$ and $B 1$ fields and application to NMR well logging. J Magn Reson 2000; 143: 12035 .
[14] Maule. J. Diploma Thesis. RWTH-Aachen. Aachen: Germany 2005.

[15] Bear J. Dynamics of fluids in porous media. New York: Dover 1988.

[16] Roth K. Soil Physics. Lecture notes. Institute of environmental physics. University of Heidelberg: Heidelberg 2007.

[17] Van Genuchten M. A closed-form equation for predicting the hydraulic conductivity of unsaturated soils. Soil Sci Soc Am J 1980; 44: 892-8.

[18] Simunek J, Sejna M, van-Genuchten M. The HYDRUS-1D software package for simulating the one-dimensional movement of water, heat, and multiple solutes in variably saturated media, version 2.0, U.S. Salinity Laboratory, Agricultural Research Service, Riverside, California USA 2008.

[19] Kastelanek F. Numerical simulation technique for vertical drainage. J Hydrol 1971; 14: 213-32.

[20] Pohlmeier A, van Dusschoten D, Weihermueller L, Schnurr U, Vereecken H. Imaging water fuxes in porous media by magnetic resonance imaging using $\mathrm{D}_{2} \mathrm{O}$ as a tracer. Magn Reson Imaging 2008; $27: 285-92$.

(C) Sucre et al.; Licensee Bentham Open.

This is an open access article licensed under the terms of the Creative Commons Attribution Non-Commercial License (http://creativecommons.org/licenses/by-nc/3.0/) which permits unrestricted, non-commercial use, distribution and reproduction in any medium, provided the work is properly cited. 